\title{
Pedicle screw placement in the lumbar spine: effect of trajectory and screw design on acute biomechanical purchase
}

\author{
Steven Wray, MD, ${ }^{1}$ Ronnie Mimran, MD, ${ }^{2}$ Sasidhar Vadapalli, MS, ${ }^{3}$ Snehal S. Shetye, $\mathrm{PhD},{ }^{4}$ \\ Kirk C. McGilvray, PhD, ${ }^{4}$ and Christian M. Puttlitz, PhD ${ }^{4}$ \\ ${ }^{1}$ Department of Neurosurgery, Atlanta Brain and Spine Care, Atlanta, Georgia; ${ }^{2}$ Department of Neurological Surgery, Pacific \\ Brain and Spine Medical Group, Danville, California; ${ }^{3}$ Research Engineering, Medtronic Spine and Biologics, Memphis, \\ Tennessee; ${ }^{4}$ Department of Mechanical Engineering and School of Biomedical Engineering, Colorado State University, Fort \\ Collins, Colorado
}

OBJECT Low bone mineral density in patients undergoing lumbar spinal surgery with screws is an especially difficult challenge because poor bone quality can severely compromise the maximum achievable purchase of the screws. A relatively new technique, the cortical bone screw trajectory, utilizes a medialized trajectory in the caudocephalad direction to engage a greater amount of cortical bone within the pars interarticularis and pedicle. The objectives of this cadaveric biomechanical study were to 1) evaluate a cortical screw system and compare its mechanical performance to the traditional pedicle screw system; 2) determine differences in bone quality associated with the cortical screw trajectory versus the normal pedicle screw insertion technique; 3) determine the cortical wall breach rate with both the cortical and traditional screw trajectories; and 4) determine the performance of the traditional screw in the cortical screw trajectory.

METHODS Fourteen fresh frozen human lumbar spine sections (L1-5) were used in this study (mean age $57 \pm 19$ years). The experimental plan involved drilling and tapping screw holes for 2 trajectories under navigation (a traditional pedicle screw and a cortical screw) in both high- and low-quality vertebrae, measuring the bone quality associated with these trajectories, placing screws in the trajectories, and evaluating the competence of the screw purchase via 2 mechanical tests (pullout and toggle). The 3 experimental variants were 1) traditional pedicle screws placed in the traditional pedicle screw trajectory, 2) traditional pedicle screws placed in the cortical screw trajectory, and 3) cortical screws placed in the cortical screw trajectory.

RESULTS A statistically significant increase in bone quality was observed for the cortical trajectories with a cortical screw $(42 \% ; p<0.001)$ and traditional pedicle screw $(48 \% ; p<0.001)$ when compared to the traditional trajectory with a traditional pedicle screw within the high-quality bone group. These significant differences were also found in the lowquality bone cohort. All mechanical parameter comparisons (screw type and trajectory) between high-quality and lowquality samples were significant $(p<0.01)$, and these data were all linearly correlated $(r \geq 0.65)$ to bone mineral density. Not all mechanical parameters determined from pullout and toggle testing were statistically significant between the 3 screw/trajectory combinations. The incidence of cortical wall breach with the cortical or traditional pedicle screw trajectories was not significantly different.

CONCLUSIONS The data demonstrated that the cortical trajectory provides denser bone that allows for utilization of smaller screws to obtain mechanical purchase that is equivalent to long pedicle screws placed in traditional pedicle screw trajectories for both normal- and low-quality bone. Overall, this biomechanical study in cadavers provides evidence that the cortical screw trajectory represents a good option to obtain fixation for the lumbar spine with low-quality bone.

http://thejns.org/doi/abs/10.3171/2014.10.SPINE14205

KEY WORDS pedicle screw; biomechanics; cortical screw; trajectory; lumbar

ABBREVIATIONS BMD $=$ bone mineral density; $\mu \mathrm{CT}=$ micro-CT .

SUBMITTED February 25, 2014. ACCEPTED October 7, 2014.

INCLUDE WHEN CITING Published online February 13, 2015; DOI: 10.3171/2014.10.SPINE14205.

DISCLOSURE Funding for this study was provided by Medtronic Spinal and Biologics as an institutional grant to Colorado State University. Drs. Wray, Mimran, and Puttlitz are consultants for Medtronic. Mr. Vadapalli is an employee of Medtronic and reports receiving clinical or research support for this study from Medtronic. 
$\mathrm{P}$ EDICLE screws are a well-established and widely accepted means for spinal fixation to treat degeneration, trauma, neoplasms, deformity, and other pathological conditions of the thoracolumbar spine..$^{3,8,15}$ Low bone mineral density (BMD) in patients presents a challenge because poor bone quality can severely compromise the maximum achievable purchase of the screws. Laboratory investigations have consistently shown that osteoporotic vertebrae demonstrate reduced insertion torque and pullout strength., ${ }^{4,5,16,19}$ Additionally, elements of screw design and placement (such as thread pitch and trajectory, respectively) can influence the screw-to-bone interface, and, ultimately, the degree of overall fixation.

Traditional pedicle screws rely on engagement of trabecular bone within both the pedicle and the vertebral body. Recently, an alternative to traditional pedicle screw trajectory fixation has been proffered in lumbar fusion constructs. This cortical bone screw trajectory utilizes a medialized trajectory in the caudocephalad direction to engage the cortical bone associated with the pars interarticularis and pedicle. A previously published cadaveric biomechanical study confirmed that the cortical screw trajectory provides significantly denser bone for screw purchase than the traditional pedicle screw trajectory. ${ }^{18}$

Previous studies have not explicitly tested the relationship between screw design and trajectory. Therefore, it remains unknown as to how the resultant construct strength is affected after placing a traditional pedicle screw in the cortical screw trajectory. Additionally, it has not been demonstrated to what extent, if any, that the presence of highor low-quality bone can have as a confounding effect on this issue. Finally, the safety (i.e., risk of neural impingement) associated with placing traditional pedicle screws in the cortical screw trajectory has not been reported. Accordingly, to address the aforementioned issues and questions, we outlined the following specific aims for the current biomechanical cadaveric study: 1) evaluate a cortical screw system and compare its mechanical performance to the traditional pedicle screw system; 2) determine differences in bone quality associated with the cortical screw trajectory versus the normal pedicle screw trajectory; 3 ) measure the cortical wall breach rate for both the cortical and pedicle screw trajectories; and 4) determine the initial stability of the pedicle screw in the cortical screw trajectory. These 4 aims were performed with both highand low-quality vertebral bone specimens to delineate the importance of bone quality on these issues.

\section{Methods}

The experimental plan involved drilling and tapping screw holes for 2 trajectories (traditional pedicle screw and cortical screw) in both normal- and low-quality vertebrae, measuring the bone quality associated with these trajectories, placing screws in the trajectories, and evaluating the competence of the screw purchase via 2 mechanical tests (pullout and toggle). Finally, correlations were performed between the mechanical test data and bone quality assessments.

\section{Specimen Sample Preparation and Allocation}

Fourteen fresh frozen human lumbar spine sections
(L1-5) were used in this study (mean age $57 \pm 19$ years; 11 females and 3 males). Extraneous soft tissue was dissected from the samples with care to not disrupt the bony and ligamentous architecture. Samples were sprayed with physiological saline at 15-minute intervals throughout the dissection process to maintain hydration. Following dissection, each lumbar spine segment (L-1 through L-5) was scanned using dual energy x-ray absorptiometry (DEXA, model Discovery A, Hologic) to determine a global, clinically relevant measure of BMD. The 7 spines with the highest overall BMD were assigned to the "high-quality" bone group, with the remaining 7 spines assigned to the "low-quality" bone group. Low-quality spines ( $n=7 ; 63 \pm$ 16 years; 6 females and 1 male) had a mean ( \pm SD) BMD of $0.470 \pm 0.088 \mathrm{~g} / \mathrm{cm}^{2}$. All of the spines in the low-quality group satisfied the WHO criteria for being "osteoporotic" (skeletal site, race, sex, and age-adjusted T-score $\leq-2.5$ ). High-quality spines $(\mathrm{n}=7 ; 52 \pm 21$ years; 5 females and 2 males) had a mean $\left( \pm\right.$ SD) BMD of $0.768 \pm 0.123 \mathrm{~g} / \mathrm{cm}^{2}$. All of the spines in the high-quality group satisfied the WHO criteria for being either "normal" (adjusted T-score $>-1.0$ ) or "osteopenic" (adjusted T-score between -1.0 and -2.5). The difference in the median BMD and T-score values between the 2 groups was statistically significant $(p<0.001$ via Mann-Whitney rank-sum test; SigmaPlot, Systat).

\section{Screw Trajectory Drilling, and Tapping}

Screw hole placement, drilling, tapping, and screw sizing were performed under navigation using a surgical imaging system (O-arm Surgical Imaging System and StealthStation S7 navigation system, Medtronic). Standard surgical techniques, tools, and practices were used throughout the surgical intervention. Bilateral screw holes were drilled and tapped in each vertebra with either a cortical or traditional pedicle trajectory (Fig. 1). Each hole was assigned to one of 2 screw system types: cortical screws or traditional pedicle screws (CD HORIZON SOLERA and LEGACY Spinal System, Medtronic). The traditional pedicle screws had 2.75 and 2.8 threads per $\mathrm{mm}$ for the 5.5- and 6.5-mmdiameter screws, respectively. Conversely, the cortical screw had a finer thread structure design (4.0 threads per $\mathrm{mm}$ for the 5.5-mm-diameter screw) for use in the cortical trajectory to obtain purchase in denser bone. Treatment allocation within a sample was based on a random number generator scheme with the following design restrictions: 1) the treatment groups were equally distributed across spinal levels and 2) the pedicle screw placement in the traditional pedicle screw trajectory was performed at all levels. This schema yielded 3 treatment groups distributed across 69 vertebral bodies (total of 138 screw holes): 1) pedicle screw in a traditional pedicle screw trajectory; 2) pedicle screw in the cortical screw trajectory; and 3) cortical screw in the cortical screw trajectory.

Cortical screw trajectory drill holes were generated line-to-line and unilaterally via the pars interarticularis and caudal to the sulcus of the facet complex. The cortical screw trajectory followed medial-to-lateral and caudalto-cephalad paths. ${ }^{18}$ The contralateral hole was prepared using the traditional pedicle screw insertion technique in which the sagittal trajectory of the screw was drilled parallel to the superior endplate of the vertebral body. Traditional pedicle screw trajectories were "undertapped" us- 


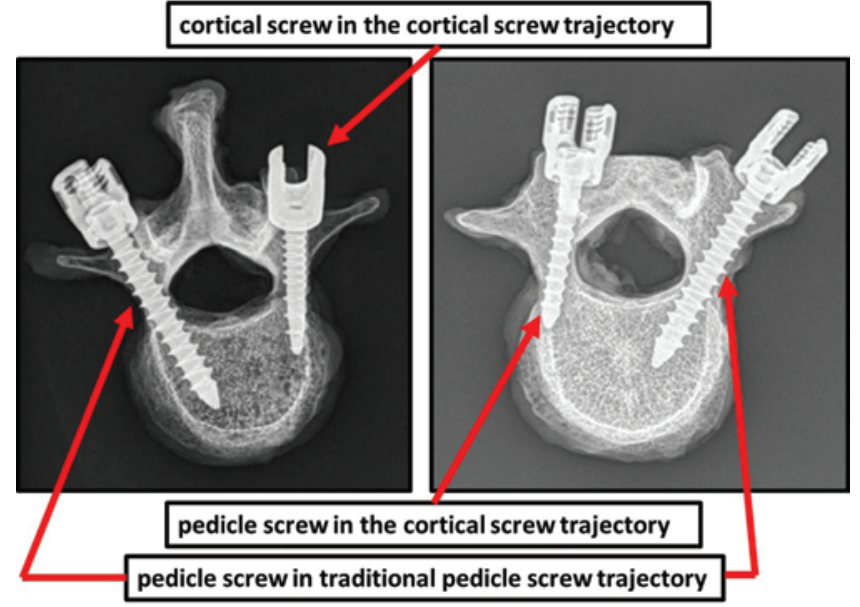

FIG. 1. Digital radiographs demonstrating the trajectories of the 3 treatment groups. The pedicle screw in the traditional pedicle trajectory variant was performed in all samples. Figure is available in color online only.

ing a cannulated tap that was $1.00 \mathrm{~mm}$ smaller than the diameter of the pedicle screw placed at that level. Cortical screw trajectories were tapped using a cannulated tap of equivalent diameter to the screw placed at that level. Screw size and length was determined using navigation data. None of the bilateral trajectories interfered with or intersected each other.

\section{Micro-CT Analysis}

To determine the bone quality and density of each local screw trajectory, each vertebral body underwent micro$\mathrm{CT}(\mu \mathrm{CT})$ scanning. Data were acquired at an isotropic resolution of $74 \mu \mathrm{m}$ (SCANCO 80, SCANCO USA, Inc.). The resulting image data were imported into a medical imaging segmentation software package (AMIRA, Visualization Sciences Group) and spatially transformed to ensure orthogonality of the image slices with respect to the principal axes of the screw holes. This protocol ensured that the resultant volume of interest was directed along the long axis of the screw. A cylindrical volume of interest was evaluated along each screw trajectory (Fig. 2 ). The minimum diameter of the evaluation volume was defined as the minor diameter of the respective screw, and the maximum diameter of the evaluation volume was defined as a 5-mm radial expansion of the minimum diameter. The evaluation length was equilibrated to the specific screw length used in each particular drilled trajectory. Thus, the volume of interest included all bone contributing to screw-bone purchase.

The voxel-based linear attenuation coefficient values reported by the $\mu \mathrm{CT}$ were converted to Hounsfield units, a more clinically relevant measure of bone quality, using the following formula ${ }^{2}: H U_{x}=\left(\mu_{x}-\mu_{w}\right) / \mu_{w} \times 1000$, where $\mu_{x}$ and $\mu_{w}$ are the linear attenuation coefficients of the given voxel and water at the effective $\mu \mathrm{CT}$ energy, respectively. All positive nonzero Hounsfield unit voxel values within the volume of interest were averaged to obtain a single Hounsfield unit value for each screw trajectory. Full 3D reconstructions of each vertebra were performed and analyzed to determine cortical wall breaches (Fig. 2).

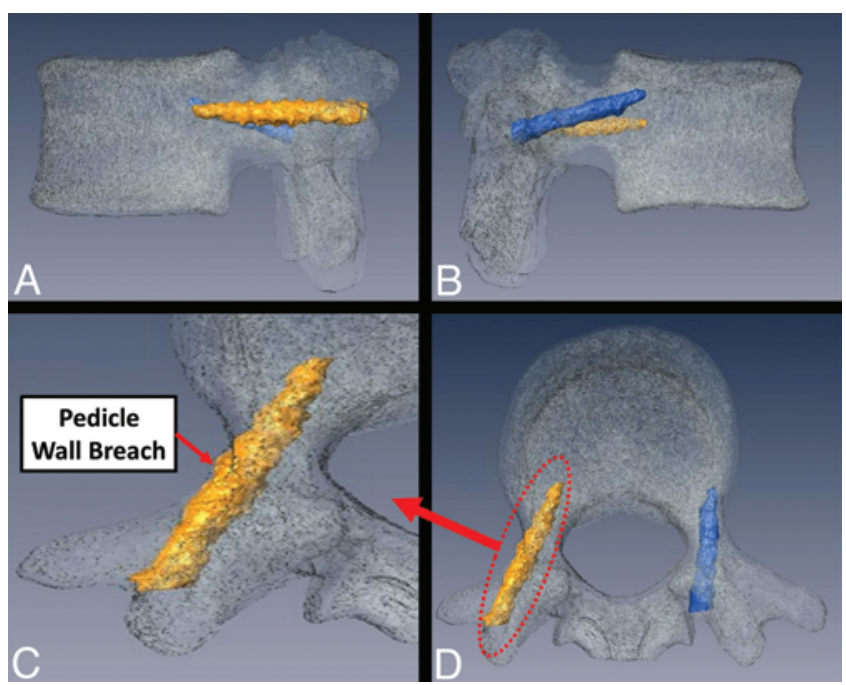

FIG. 2. Lateral ( $A$ and $B$ ) and anterior ( $C$ and $D$ ) views illustrating drilled hole volumes along the traditional (orange) and cortical (blue) trajectories. The traditional trajectory is primarily oriented in the anteromedial direction, whereas the cortical trajectory projects in the anteromediolateral direction. A sample reconstruction indicating a breach (depicted in $\mathrm{C}$ and $\mathrm{D}$ ) in the lateral wall of the pedicle. Figure is available in color online only.

Following $\mu \mathrm{CT}$ scanning, the appropriate screws in terms of type, diameter, and length (based on navigation data during the aforementioned drilling and tapping procedure) were placed in their drilled and tapped holes. Digital radiographs after screw insertion were obtained to document trajectory and proper placement.

\section{Biomechanical Testing}

The acute biomechanical integrity of the screw-bone interface was determined using standard screw pullout and screw toggle testing techniques. ${ }^{18}$ Testing allocation for each of the 69 lumbar vertebrae was based on a random number generator scheme with the following design restrictions: 1) the treatment groups were equally distributed across testing methods (pullout and toggle), and 2) an individual vertebra from each spinal section (L1-5) had a minimum of 1 vertebral body allocated to each test. Thirty-five vertebral bodies were allocated to pullout testing, yielding 70 individual screw pullout samples. Thirty-four vertebral bodies were allocated to toggle testing, yielding 68 individual toggle tests.

For screw pullout tests, the screw of interest was quasistatically extracted while the vertebral body was rigidly constrained. A driver, specific to the screw of interest, was used to couple the vertebral body to the actuator of the materials testing machine (MiniBionix 858, MTS). Uniaxial alignment of the MTS actuator, attached force transducer (5 kN, MTS), and the screw placement was accomplished using an adjustable, multiaxial vice. The screw rigidly attached to the surgical driver was withdrawn uniaxially at a rate of $10 \mathrm{~mm} / \mathrm{min} .{ }^{17,18,20}$ Failure of the screw-bone interface was defined as a decrease in the monotonically increasing force profile and/or if there was clearly observable bone failure. After failure, the contralateral screw was 
tested in an identical fashion. Failure force and construct stiffness were calculated from the resultant force displacement data. Stiffness was defined as the slope of the linear region of the uniaxial force displacement curve.

Screw toggle testing was implemented to provide a more clinically relevant failure scenario (mixed loading regimen) than traditional pullout testing. ${ }^{18}$ Specifically, pedicle screws are exposed to simultaneous combinations of bending moments, shear stresses, and axial stresses in multiple planes in vivo. ${ }^{18}$ Therefore, to more closely mimic the in vivo environment, a series of screw-rod toggle tests was performed. In this test, screws were rigidly attached to a surgical driver and collinearly aligned with the MTS actuator. The vertebral body was rigidly constrained to a 6-df load cell (model 610, AMTI) using mechanical grips. The surgical driver was then removed, and a longitudinal rod was attached to the screw head that served as a lever arm to apply a mixed loading regimen to the screw. An adjustable $X-Y$ table was used to ensure a constant lever arm of $40 \mathrm{~mm}$. The free end of the rod was rigidly attached to the MTS actuator and uniaxially withdrawn at a rate of $10 \mathrm{~mm} / \mathrm{min}$. Three-dimensional shear, moment, and displacement data were resolved into resultant moment and force magnitudes. Failure of the rod-screw-bone construct was established as a decrease in the monotonically increasing force profile and/or if there was a clearly observable bone or hardware failure. After failure, the contralateral screw-rod construct was tested in an identical fashion. Failure force (maximum force achieved in the monotonically increasing force-displacement pullout data), failure moment (maximum resultant moment achieved in the monotonically increasing resultant moment-displacement toggle data), and construct stiffness (slope within the linear portion of the force-displacement pullout data) were calculated from the resultant force displacement and moment data. In addition, the mechanical data were correlated with the previous $\mu \mathrm{CT}$ calculations of bone quality to determine the relationship between bone quality and screw toggle performance.

A 1-way ANOVA was performed on the mechanical test data (screw pullout and toggle) to determine statistical significance between treatment groups (SigmaPlot version 11.0, Systat Software Inc.); p values $<0.05$ were considered to be statistically significant. The mechanical data were correlated with the $\mu \mathrm{CT}$ density measurements of bone quality using a standard Pearson's correlation coefficient.

\section{Results \\ Screw Geometry}

The screw diameter and length information are summarized in Table 1. On average, the pedicle screws placed in the traditional pedicle trajectory had significantly greater core diameters than cortical screws in the cortical trajectory $(46 \%, \mathrm{p}<0.001)$ and pedicle screws in the cortical trajectory $(47 \%, \mathrm{p}<0.001)$ (Table 1$)$. On average, the pedicle screws placed in the traditional pedicle trajectory were significantly longer than cortical screws in the cortical trajectory (by an average of $18 \%, \mathrm{p}<0.01$ ) and pedicle screws in the cortical trajectory $(19 \%, \mathrm{p}<0.001)$ (Table 1). There were no statistically significant differences ob- served either in diameter or length between the cortical screw in the cortical trajectory and the pedicle screw in the cortical trajectory. Overall, the cortical trajectory requires a significantly smaller diameter and shorter screw than a traditional pedicle trajectory.

\section{Micro-CT Analysis}

Micro-CT data were used to determine differences in bone quality associated with the cortical screw trajectory versus the traditional pedicle screw trajectory, and to detect the possible risk of cortical wall breach with both the cortical and traditional screw trajectories. Within the high-quality $(p=0.55)$ and low-quality $(p=0.27)$ bone groups, no statistical differences in bone density were observed between the cortical trajectories, regardless of screw type (cortical or pedicle; Fig. 3). A statistically significant increase in bone quality was observed for the cortical trajectories with a cortical screw $(42 \%$; $\mathrm{p}<0.001)$ and pedicle screw $(48 \% ; \mathrm{p}<0.001)$ when compared to the traditional pedicle trajectory with a pedicle screw within the high-quality bone group. These significant differences $(\mathrm{p}<0.01)$ were also found in the low-quality bone cohort. The bone quality for the cortical trajectories within the high-quality group was statistically greater than that for any trajectory in the low-quality group $(\mathrm{p}<0.001)$. The bone quality of the traditional pedicle trajectory in the high-quality group was not significantly different from that of the cortical trajectory for the cortical and pedicle screws within the low-quality group $(\mathrm{p}=0.95$ and 0.38 , respectively). The bone quality for all trajectories within the high-quality group was statistically greater than that for the same trajectories in the low-quality group $(\mathrm{p}<0.001)$. Overall, the data indicate that the BMD (i.e., bone quality) associated with the cortical trajectory is significantly greater than the traditional pedicle trajectory regardless of screw type used or the overall quality (low quality or high quality) of the bone.

Three-dimensional reconstructions (Fig. 2) based on $\mu \mathrm{CT}$ data were used to visually determine a possible risk of cortical wall breach for the traditional and both cortical trajectories. The data indicate an $11 \%$ incidence of cortical wall breach across the entire specimen population (Table 2). The incidence of cortical breach with either the cortical or traditional pedicle screw trajectories was not significantly different.

\section{Biomechanical Analyses}

For the pullout tests, neither screw profile (pedicle or cortical) nor screw hole trajectory (pedicle or cortical) had a statistically significant effect $(\mathrm{p}=0.63)$ on ultimate failure load in high-quality bone (Fig. 4). These results were mirrored for the low-quality cohort $(p=0.90)$. All comparisons (screw type and trajectory) of screw pullout force between high-quality and low-quality samples were significant $(\mathrm{p} \leq 0.01)$. No statistically significant differences were noted for pullout stiffness in the high-quality $(\mathrm{p}=$ $0.34)$ and low-quality $(p=0.64)$ samples. All comparisons of stiffness (for screw type and trajectory) calculated from pullout testing between high-quality and low-quality samples were significant ( $\mathrm{p} \leq 0.02$; Fig. 5). 
TABLE 1. Descriptive statistics of screw geometries utilized for pullout and toggle testing*

\begin{tabular}{|c|c|c|c|c|c|c|c|}
\hline \multicolumn{2}{|c|}{ Sample Description } & \multicolumn{3}{|c|}{ Pullout Testing } & \multicolumn{3}{|c|}{ Toggle Testing } \\
\hline Trajectory & Screw Type & $\begin{array}{l}\text { Screw Length } \\
(\mathrm{mm})\end{array}$ & $\begin{array}{c}\text { Screw Diameter } \\
(\mathrm{mm})\end{array}$ & $\begin{array}{c}\text { No. of } \\
\text { Samples }\end{array}$ & $\begin{array}{l}\text { Screw Length } \\
(\mathrm{mm})\end{array}$ & $\begin{array}{c}\text { Screw Diameter } \\
(\mathrm{mm})\end{array}$ & $\begin{array}{c}\text { No. of } \\
\text { Samples }\end{array}$ \\
\hline \multicolumn{8}{|c|}{ High-quality bone } \\
\hline Cortical & Cortical & $30.00 \pm 0.00^{A}$ & $5.28 \pm 0.36^{c}$ & 9 & $29.38 \pm 1.78$ & $5.25 \pm 0.37^{\mathrm{K}}$ & 8 \\
\hline Cortical & Pedicle & $29.38 \pm 1.77^{B}$ & $5.25 \pm 0.46^{\mathrm{D}}$ & 8 & $30.63 \pm 3.20^{J}$ & $5.23 \pm 0.52^{L}$ & 9 \\
\hline Pedicle & Pedicle & $43.82 \pm 3.86^{A, B}$ & $6.31 \pm 0.40^{C, D}$ & 17 & $45.31 \pm\left. 3.86\right|^{J}$ & $6.31 \pm 0.40^{\mathrm{K}, \mathrm{L}}$ & 17 \\
\hline \multicolumn{8}{|c|}{ Low-quality bone } \\
\hline Cortical & Cortical & $29.44 \pm 4.64^{\mathrm{E}}$ & $5.27 \pm 0.36^{G}$ & 9 & $28.75 \pm 2.31^{\mathrm{M}}$ & $5.31 \pm 0.37^{\circ}$ & 8 \\
\hline Cortical & Pedicle & $27.78 \pm 2.64^{F}$ & $5.17 \pm 0.50$ & 9 & $28.75 \pm 2.31^{\mathrm{N}}$ & $5.25 \pm 0.46^{P}$ & 9 \\
\hline Pedicle & Pedicle & $40.31 \pm 4.64^{E, F}$ & $6.13 \pm 0.50^{\mathrm{G}, \mathrm{H}}$ & 18 & $41.76 \pm 4.30^{\mathrm{M}, \mathrm{N}}$ & $6.08 \pm 0.51^{0, P}$ & 17 \\
\hline
\end{tabular}

* Mean values are presented as the mean \pm SD. Like letters indicate statistically significant differences between groups $(p<0.01$ for all letters).

No statistically significant differences were noted in the failure force $(p=0.67)$, stiffness $(p=0.57)$, and failure moment $(\mathrm{p}=0.41)$ during toggle testing in the highquality samples (Table 3 ). In addition, no statistically significant differences were noted in the failure force $(\mathrm{p}=$ $0.27)$, stiffness $(\mathrm{p}=0.19)$, and failure moment $(\mathrm{p}=0.69)$ during toggle testing in the low-quality samples (Table 3). All comparisons of failure force, stiffness, and failure moment for equivalent treatment types (screw type and trajectory) between high-quality and low-quality samples under toggle testing were significantly different $(\mathrm{p} \leq 0.01$ for all comparisons).

\section{Correlations Between Bone Quality and Mechanical Parameters}

Correlations between screw pullout force and bone quality were observed for the pedicle screw in the cortical trajectory $(r=0.91 ; p<0.01)$, the pedicle screw in the pedicle trajectory $(r=0.92 ; p<0.01)$, and the cortical screw in the cortical trajectory $(r=0.73 ; p=0.01)$. These data indicate that there is a statistical likelihood that pullout failure load and bone quality increases are linearly correlated for

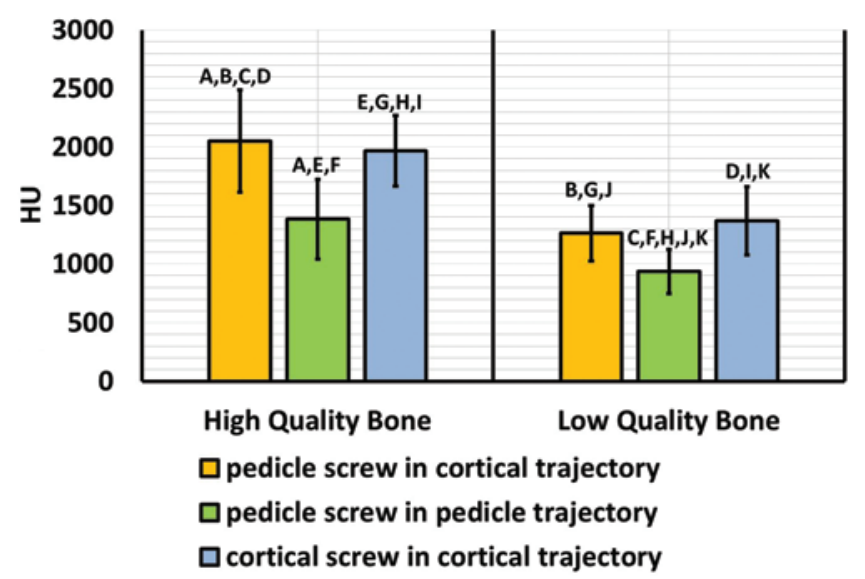

FIG. 3. Bone quality, based on $\mu \mathrm{CT}$ data and transformed into Hounsfield units (HU, y axis), associated with the cortical screw trajectory versus the normal pedicle screw trajectory. Means are shown with SD (error bars). Like letters indicate statistically significant differences: $p<$ 0.01 for all comparisons. Figure is available in color online only. all treatment groups. In addition, high correlations between screw toggle failure force and bone quality were observed for the pedicle screw in the cortical trajectory ( $\mathrm{r}$ $=0.74 ; \mathrm{p}<0.01)$, the pedicle screw in the pedicle trajectory $(r=0.79 ; p<0.01)$, and the cortical screw in the cortical trajectory $(r=0.72 ; p<0.01)$. Finally, positive correlations between screw toggle failure force and bone quality were observed for the pedicle screw in the cortical trajectory $(\mathrm{r}$ $=0.65 ; \mathrm{p}=0.005)$, the pedicle screw in the pedicle trajectory $(\mathrm{r}=0.77 ; \mathrm{p}<0.01)$, and the cortical screw in the cortical trajectory $(\mathrm{r}=0.65 ; \mathrm{p}<0.01)$.

\section{Discussion}

The patient who requires posterior spinal instrumentation and has reduced bone quality represents an especially difficult challenge for the attending clinician. Laboratory data have consistently demonstrated that significantly reduced pullout strength and insertion torque are seen in these patients, ${ }^{4,5,9,16,19}$ resulting in a lower probability of obtaining rigid fixation and a solid arthrodesis. There have been a number of different techniques and technologies that have been proposed for obtaining better screw purchase in low-quality bone. , $, 6,7,10,12-14$ Most of these novel techniques use the traditional pedicle trajectory and inject bone cement into the drilled screw hole and/or use a coating on the screw surface to promote better hardware purchase. However, these methods suffer from (at least partial) reliance on the existing reduced trabecular bone structure to obtain and maintain competent screw-to-bone fixation. On the contrary, in the current cadaveric biomechanical study, the cortical screw trajectory had an inher-

TABLE 2. Cortical wall breach data as observed from $\mu C T$ 3D reconstructions

\begin{tabular}{cccc}
\hline Trajectory & $\begin{array}{c}\text { Total No. of } \\
\text { Breaches }\end{array}$ & $\begin{array}{c}\text { Total No. of } \\
\text { Samples }\end{array}$ & Breach \\
\hline Cortical & & & \\
\hline Cortical screw & 4 & 35 & $11.4 \%$ \\
\hline $\begin{array}{c}\text { Pedicle screw } \\
\text { Pedicle }\end{array}$ & 3 & 34 & $8.8 \%$ \\
\hline Pedicle screw & 9 & 69 & $13.0 \%$ \\
\hline
\end{tabular}




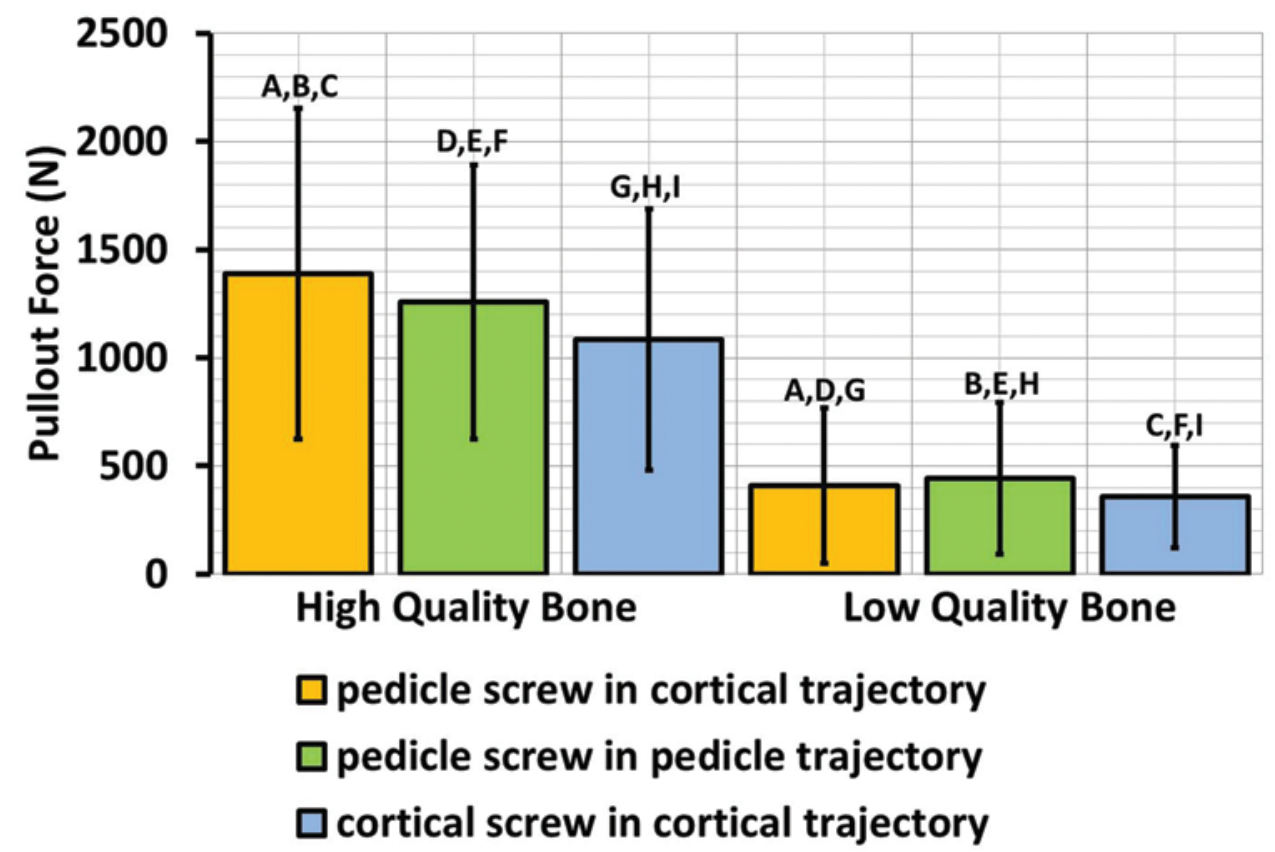

FIG. 4. Failure force measured during destructive screw pullout testing. Means are shown with SD (error bars). Like letters indicate statistically significant differences: $p<0.01(\mathrm{~A}-\mathrm{F}$ and $\mathrm{H})$ and $p=0.01(\mathrm{G})$. No statistically significant differences were detected in the data within the high-quality groups $(p=0.63)$ or within the low-quality groups $(p=0.90)$. Figure is available in color online only.

ently greater bone density along its path. This trajectory has a theoretical advantage in osteoporotic patients whose trabecular bone may be resorbed over cortical bone; thus, achieving cortical purchase may be preferred by the surgeon.

The current cadaveric biomechanical study investigated and compared traditional pedicle screw fixation of the lumbar spine with that of more novel cortical screw trajectory in both low-quality (analogous to osteoporotic) and high-quality (analogous to nonosteoporotic) vertebrae. Rigid fixation using pedicle screws in the pedicle screw trajectory was compared with that of pedicle screws and cortical screws (which have a different thread pitch geometry) in the cortical trajectory. As expected, the data indi-

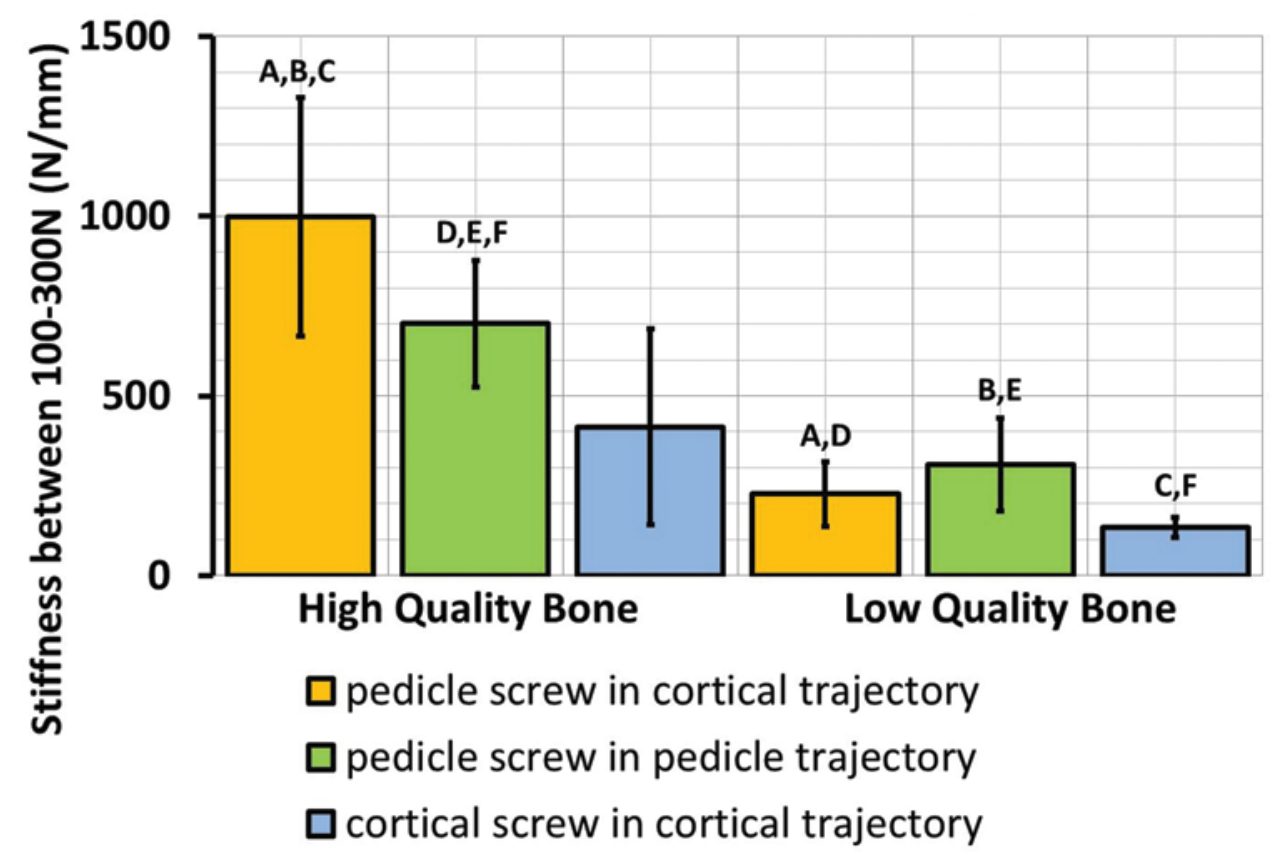

FIG. 5. Stiffness calculated during destructive screw pullout testing. Means are shown with SD (error bars). Like letters indicate statistically significant differences: $p<0.01(A, B, E$, and $F), p=0.01(C)$, and $p=0.02(D)$. No statistically significant differences were detected in the data within the high-quality groups $(p=0.34)$ or within the low-quality groups $(p=0.64)$. Figure is available in color online only. 
TABLE 3. Outcome parameters calculated from failure toggle testing of bone-screw-rod constructs*

\begin{tabular}{|c|c|c|c|c|c|}
\hline \multicolumn{2}{|c|}{ Parameter } & \multicolumn{2}{|c|}{ High-Quality Bone } & \multicolumn{2}{|c|}{ Low-Quality Bone } \\
\hline Trajectory & Screw Type & Value & $p$ Value & Value & $\mathrm{p}$ Value \\
\hline \multicolumn{6}{|c|}{ Resultant failure force $(\mathrm{N})$} \\
\hline Cortical & Cortical & $909.15 \pm 127.94$ & \multirow{3}{*}{0.67} & $557.69 \pm 115.96$ & \multirow{3}{*}{0.27} \\
\hline Cortical & Pedicle & $767.84 \pm 128.85$ & & $314.60 \pm 128.85$ & \\
\hline Pedicle & Pedicle & $807.50 \pm 71.01$ & & $363.29 \pm 57.73$ & \\
\hline \multicolumn{6}{|l|}{ Stiffness (N/mm) } \\
\hline Cortical & Cortical & $70.96 \pm 7.00$ & \multirow{3}{*}{0.57} & $24.43 \pm 4.92$ & \multirow{3}{*}{0.19} \\
\hline Cortical & Pedicle & $57.04 \pm 10.69$ & & $45.55 \pm 8.48$ & \\
\hline Pedicle & Pedicle & $61.79 \pm 6.17$ & & $32.87 \pm 5.35$ & \\
\hline \multicolumn{6}{|c|}{ Resultant Failure Moment (N-m) } \\
\hline Cortical & Cortical & $15.01 \pm 2.52$ & \multirow{3}{*}{0.41} & $5.66 \pm 1.41$ & \multirow{3}{*}{0.69} \\
\hline Cortical & Pedicle & $15.03 \pm 2.93$ & & $5.40 \pm 1.74$ & \\
\hline Pedicle & Pedicle & $11.46 \pm 1.74$ & & $4.46 \pm 0.79$ & \\
\hline
\end{tabular}

* No statistically significant differences were detected in the data for comparisons within a specific sample group (high- and low-quality bone).

cate that decreased bone quality adversely affected pullout and toggle force. Ultimate failure load and stiffness obtained from pullout testing were not statistically different based on screw trajectory or screw profile in osteoporotic and nonosteoporotic bone. Similarly, ultimate failure load, failure moment, and construct stiffness during screw toggle testing were not significantly different with screw profile or trajectory as applied to both osteoporotic and nonosteoporotic bone. Bone mineral density, as measured using high-fidelity $\mu \mathrm{CT}$, along the cortical screw trajectory was significantly greater than along the pedicle screw trajectory regardless of thread pitch or overall BMD (as measured using dual energy x-ray absorptiometry to delineate high and low quality bone) of the specimen. Denser bone in the cortical screw trajectory required significantly smaller-diameter and shorter screws than pedicle screw fixation. A 6.5-mm-diameter $\times 40$-mm-long pedicle screw in the traditional pedicle screw trajectory has $853 \mathrm{~mm}^{2}$ of surface area available for purchase with bone, whereas the 5.5-mm-diameter $\times 30$-mm-long cortical screw (the most common size used in the current cadaveric biomechanical study) has $546 \mathrm{~mm}^{2}$ of available surface area (a $36 \%$ reduction compared with the pedicle screw). Given that these screws demonstrated statistical equivalence in mechanical performance, it can be concluded that the holding strength (force/area) afforded by the cortical screw trajectory is inherently greater and provides better screw fixation.

The inherent risk of the pedicle trajectory is a breach of the cortical wall and potential impingement of the perispinal neural elements. In the current study, the risk of a cortical breach was not statistically significantly different using either trajectory or screw profile. A range of $8.8 \%-13 \%$ of the experimental variants exhibited some degree of cortical wall breach. These data are consistent with previously reported clinical breach rates of approximately $12 \% .{ }^{11} \mathrm{~A}$ previous laboratory study, which did not use navigation, reported an overall cortical wall breach rate of approximately $20 \%$ using the cortical trajectory. ${ }^{18}$ The use of navigation in the current cadaveric biomechanical study reduced the incidence of cortical wall breach to $11.4 \%$ in this experimental cohort, an occurrence level that falls within the previously reported range for pedicle screws. Based on the current cadaveric biomechanical study results and with the medial-to-lateral trajectory of the cortical screw, the use of cortical screws could lead to a decrease in the impingement of the perispinal neural elements.

The findings of this cadaveric biomechanical study should be held within the context of its limitations. Most notably, the data reported herein represent the expected mechanical behavior of the different experimental variants in the acute (i.e., immediately postoperative) time frame. Only a properly designed fatigue study can shed light on the long-term behavior of these screw/trajectory combinations. Of course, given the cadaveric nature of the current study, performance of high cycle fatigue (i.e., greater than 30,000 cycles) would have introduced the significantly confounding effect of temporal specimen degradation. In addition, one main objective of the current study was to examine the effect of bone density differences associated with the 2 different screw trajectories and thus eliminated the possibility of using synthetic test platforms. The coupling of these 2 issues, specimen degradation and inability to use synthetic materials, presents a nearly intractable scenario for designing a high cycle fatigue study.

\section{Conclusions}

The current cadaveric biomechanical study demonstrated that the cortical trajectory aligned the screws with denser bone. Equivalent mechanical fixation was observed between the shorter cortical screws and traditional pedicle screws using both pullout and toggle evaluations. These data were consistent for both normal- and low-quality bone.

\section{References}

1. Bai C, Shao L, Da Silva AJ, Zhao Z: A generalized model for the conversion from CT numbers to linear attenuation coefficients. IEEE Trans Nucl Sci 50:1510-1515, 2003

2. Chang MC, Kao HC, Ying SH, Liu CL: Polymethylmethacrylate augmentation of cannulated pedicle screws for fixation in osteoporotic spines and comparison of its clinical results 
and biomechanical characteristics with the needle injection method. J Spinal Disord Tech 26:305-315, 2013

3. Cheng LM, Wang JJ, Zeng ZL, Zhu R, Yu Y, Li C, et al: Pedicle screw fixation for traumatic fractures of the thoracic and lumbar spine. Cochrane Database Syst Rev 5:CD009073, 2013

4. Cook SD, Salkeld SL, Stanley T, Faciane A, Miller SD: Biomechanical study of pedicle screw fixation in severely osteoporotic bone. Spine J 4:402-408, 2004

5. Davne SH, Myers DL: Complications of lumbar spinal fusion with transpedicular instrumentation. Spine (Phila Pa 1976) 17 (6 Suppl):S184-S189, 1992

6. Frankel BM, Jones T, Wang C: Segmental polymethylmethacrylate-augmented pedicle screw fixation in patients with bone softening caused by osteoporosis and metastatic tumor involvement: a clinical evaluation. Neurosurgery 61:531538, 2007

7. Fransen P: Increasing pedicle screw anchoring in the osteoporotic spine by cement injection through the implant. Technical note and report of three cases. J Neurosurg Spine 7:366-369, 2007

8. Gaines RW Jr: The use of pedicle-screw internal fixation for the operative treatment of spinal disorders. J Bone Joint Surg Am 82-A:1458-1476, 2000

9. Gilbert SG, Johns PC, Chow DC, Black RC: Relation of vertebral bone screw axial pullout strength to quantitative computed tomographic trabecular bone mineral content. J Spinal Disord 6:513-521, 1993

10. Hasegawa T, Inufusa A, Imai Y, Mikawa Y, Lim TH, An HS Hydroxyapatite-coating of pedicle screws improves resistance against pull-out force in the osteoporotic canine lumbar spine model: a pilot study. Spine J 5:239-243, 2005

11. Hsu K, Zucherman JF, White AH, Wynne G: Internal fixation with pedicle screws, in White AH, Rothman RH, Ray CD (eds): Lumbar Spine Surgery. St. Louis: Mosby, 1987, pp 322-338

12. Jiang L, Arlet V, Beckman L, Steffen T: Double pedicle screw instrumentation in the osteoporotic spine: a biomechanical feasibility study. J Spinal Disord Tech 20:430-435, 2007

13. Kettler A, Schmoelz W, Shezifi Y, Ohana N, Ben-Arye A, Claes L, et al: Biomechanical performance of the new BeadEx implant in the treatment of osteoporotic vertebral body compression fractures: restoration and maintenance of height and stability. Clin Biomech (Bristol, Avon) 21:676682,2006
14. Liu D, Zhang Y, Zhang B, Xie QY, Wang CR, Liu JB, et al: Comparison of expansive pedicle screw and polymethylmethacrylate-augmented pedicle screw in osteoporotic sheep lumbar vertebrae: biomechanical and interfacial evaluations. PLoS ONE 8:e74827, 2013

15. McCormick J, Aebi M, Toby D, Arlet V: Pedicle screw instrumentation and spinal deformities: have we gone too far? Eur Spine J 22 (Suppl 2):S216-S224, 2013

16. Okuyama K, Abe E, Suzuki T, Tamura Y, Chiba M, Sato K: Influence of bone mineral density on pedicle screw fixation: a study of pedicle screw fixation augmenting posterior lumbar interbody fusion in elderly patients. Spine J 1:402-407, 2001

17. Renner SM, Lim TH, Kim WJ, Katolik L, An HS, Andersson GB: Augmentation of pedicle screw fixation strength using an injectable calcium phosphate cement as a function of injection timing and method. Spine (Phila Pa 1976) 29:E212E216, 2004

18. Santoni BG, Hynes RA, McGilvray KC, Rodriguez-Canessa G, Lyons AS, Henson MA, et al: Cortical bone trajectory for lumbar pedicle screws. Spine J 9:366-373, 2009

19. Suzuki T, Abe E, Okuyama K, Sato K: Improving the pullout strength of pedicle screws by screw coupling. J Spinal Disord 14:399-403, 2001

20. Yazu M, Kin A, Kosaka R, Kinoshita M, Abe M: Efficacy of novel-concept pedicle screw fixation augmented with calcium phosphate cement in the osteoporotic spine. J Orthop Sci 10:56-61, 2005

\section{Author Contributions}

Conception and design: Puttlitz, Wray, Mimran, Vadapalli. Acquisition of data: Puttlitz, Wray, Vadapalli, Shetye, McGilvray. Analysis and interpretation of data: Puttlitz, Vadapalli, Shetye. Drafting the article: Puttlitz, Wray, Vadapalli. Critically revising the article: Puttlitz, Wray, Mimran, Vadapalli. Reviewed submitted version of manuscript: all authors. Approved the final version of the manuscript on behalf of all authors: Puttlitz. Statistical analysis: Puttlitz. Study supervision: Puttlitz.

\section{Correspondence}

Christian M. Puttlitz, Department of Mechanical Engineering, School of Biomedical Engineering, Colorado State University, 1374 Campus Delivery, Colorado State University, Fort Collins, CO 80523-1374. email: puttlitz@engr.colostate.edu. 